\title{
MULTICHANNEL ESTIMATION OF COIL SENSITIVITIES IN PARALLEL MRI
}

\author{
Robert L. Morrison, $\mathrm{Jr}^{\dagger}$, Mathews Jacob ${ }^{*}$, and Minh N. Do ${ }^{\dagger *}$ \\ Department of Electrical and Computer Engineering \\ ${ }^{\dagger}$ Coordinated Science Laboratory and *Beckman Institute \\ University of Illinois at Urbana-Champaign \\ rlmorris@uiuc.edu, mjacob@uiuc.edu, minhdo@uiuc.edu
}

\begin{abstract}
We consider the problem of estimating receiver coil sensitivity functions in parallel MRI. By exploiting the multichannel nature of the problem, where multiple acquisitions of the same image function are obtained with different sensitivity weightings, we obtain a subspacebased framework for directly solving for the sensitivity functions. The proposed approach does not rely on the sum-of-squares assumption used in existing estimation schemes; this assumption tends to be violated towards the center of the image, thus leading to errors in the sensitivity estimates. Our approach eliminates this problem, producing superior sensitivity estimates in comparsion to the sum-ofsquares technique. In addition, the proposed restoration procedure is non-iterative, computationally efficient, and applicable both to cases where pilot scans are available or where auto-calibration data are collected with each scan. We present experimental results using actual and simulated data to assess the performance of our approach in comparison with existing methods.
\end{abstract}

Index Terms - parallel MRI, multichannel deconvolution, image restoration

\section{INTRODUCTION}

Parallel Magnetic Resonance Imaging (PMRI) is a fast MRI method where multiple aquisitions of subsampled $k$-space data are obtained simultaneously from multiple coils with different geometric orientations. The image function can be recovered from the subsampled data given knowledge of the sensitivity functions associated with each coil [1].

In general, the coil sensitivity functions are not known, and must be estimated from the measured data. One approach is to perform the sensitivity estimation in image space; this is the approach taken in SENSE [1]. Another approach is to perform the estimation in $k$ space, which is the approach used in SMASH [2] and GRAPPA [3]. Both of these approaches make sum-of-squares (SOS) assumptions (defined in the next section). In brain imaging (circular coil geometry), these assumptions are violated towards the center of the image, thus leading to errors in the sensitivity estimates. This leads to potential problems such as aliasing artifacts and increase in g-factor.

We propose a new approach to the coil sensitivity estimation that does not make the SOS assumptions, thus eliminating the above mentioned potential problems. In our approach, we exploit the multichannel nature of the coil sensitivity estimation problem, where the sensitivity-weighted images are related to the common image function through a bank of parallel channel responses (i.e., the unknown

This work was supported by the National Science Foundation under Grant CCR 0430877.

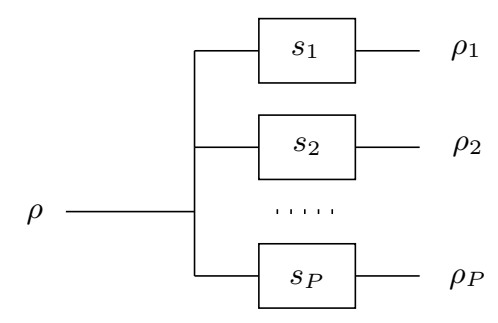

Fig. 1. Diagram illustrating the multichannel nature of the coil sensitivity problem. Here, $\rho[n, m]$ is the image function, $\left\{s_{i}[n, m]\right\}$ are the coil sensitivity functions, and $\left\{\rho_{i}[n, m]\right\}$ are the sensitivityweighted images, where $\rho_{i}[n, m]=\rho[n, m] s_{i}[n, m]$ (i.e., the filtering operation is described by multiplication in the spatial domain).

sensitivity functions), as shown in Figure 1. Here, the output signals $\left\{\rho_{i}[n, m]\right\}$ are known, but the common input $\rho[n, m]$ and channel responses $\left\{s_{i}[n, m]\right\}$ are both unknown. Thus, the coil sensitivity estimation problem has a similar flavor to the more general problem of blind multichannel deconvolution (BMD). For the BMD problem, subspace-based techniques such as EVAM have been proposed that determine the unknown FIR coefficients of the channels using a linear algebraic framework $[4,5]$.

A distinction between our problem and the BMD problem is that the filtering operation considered here is described by multiplication in the spatial domain: $\rho_{i}[n, m]=\rho[n, m] s_{i}[n, m]$. It is possible to pose the sensitivity estimation problem as the convolution of a small number of coefficients in the Fourier domain (i.e., $\hat{\rho}_{i}\left[k_{n}, k_{m}\right]=\hat{\rho}\left[k_{n}, k_{m}\right] * \hat{s}_{i}\left[k_{n}, k_{m}\right]$ ). However, the sensitivity functions are discontinuous at the edges of the image, and thus, the Fourier basis provides a poor representation of these functions. Therefore, instead of using EVAM directly, we propose a novel EVAM-like technique for estimating the coil sensitivity functions. To deal with the spatial-domain multiplication, we represent the unknown sensitivity functions using a polynomial basis expansion. This allows the polynomial coefficients of the coil sensitivity functions to be determined through a linear algebraic formulation similar to EVAM. Aided by the sensitivity estimates, SENSE is used to reconstruct the image function.

Our approach is applicable both to cases where (a) pilot scans are available and (b) auto-calibration data are collected at the center of $k$-space with each scan. In the later case, the sensitivity estimation is performed using low-resolution images formed from the auto-calibration data. In addition, the proposed technique is non- 
iterative, in contrast to [6], and has a computationally efficient form. We present experimental results using actual and simulated PMRI data to assess the performance of the proposed approach. The results show that the sensitivity estimates from our approach can produce superior image restorations using SENSE in comparison to the standard SOS estimates. The performance of our method is evaluated using different acceleration factors and auto-calibration data sizes.

\section{PROBLEM FORMULATION}

In parallel MRI, the $k$-space data are acquired simultaneously from $P$ different receiver coils according to the equation

$$
\hat{\rho}_{i}\left(\boldsymbol{k}_{l}\right)=\int \rho(\boldsymbol{r}) s_{i}(\boldsymbol{r}) e^{j 2 \pi \boldsymbol{k}_{l} \cdot \boldsymbol{r}} d \boldsymbol{r},
$$

where $\rho(\boldsymbol{r})$ is the image function to be recovered, $\hat{\rho}_{i}\left(\boldsymbol{k}_{l}\right)$ and $s_{i}(\boldsymbol{r})$ are the $k$-space data and sensitivity functions for the $i$-th coil $(i=$ $1,2, \ldots, P)$, respectively, $\boldsymbol{r}=(x, y)$ are spatial-domain coordinates, and $\boldsymbol{k}_{l}=\left(k_{x}[l], k_{y}[l]\right)(1 \leq l \leq L)$ are samples in $k$-space. Note that (1) is the Fourier transform of the sensitivity-weighted images $\rho_{i}(\boldsymbol{r}) \stackrel{\text { def }}{=} \rho(\boldsymbol{r}) s_{i}(\boldsymbol{r})$. The goal of parallel MRI image reconstruction is to recover $\rho(\boldsymbol{r})$ from $\hat{\rho}_{i}\left(\boldsymbol{k}_{l}\right)$, where the $k$-space data are undersampled with respect to the Nyquist condition.

The sensitivity functions $s_{i}(\boldsymbol{r})$ are generally not known; they must be estimated from either initial reference measurements or from auto-calibration data collected with each scan. In many applications, it is feasible to acquire a small amount of auto-calibration data at the center of $k$-space sampled at the Nyquist rate. This data can be used to form low-resolution unaliased images for each coil to estimate the sensitivity functions. These low-resolution images can be modeled as

$$
\rho_{i}[n, m]=\rho[n, m] s_{i}[n, m],
$$

where $n=0,1, \ldots, N-1$ and $m=0,1, \ldots, M-1$ are spatialdomain coordinates for the $x$ and $y$ dimensions, respectively. Here, the auto-calibration data are zero padded prior to performing an inverse Fourier transform; the dimensions of the auto-calibration data $N^{\prime}, M^{\prime}$ satisfy $N^{\prime} \leq N$ and $M^{\prime} \leq M$. Given $\rho_{i}[n, m]$ in (2), estimates of the sensitivity functions can be obtained using the sum-ofsquares (SOS) technique, where it is assumed that $\rho[n, m]$ is realvalued and the squared sensitivity functions sum to a constant $\beta$, so that $\sqrt{\sum_{i}\left|\rho_{i}[n, m]\right|^{2}}=\rho[n, m] \sqrt{\sum_{i}\left|s_{i}[n, m]\right|^{2}} \approx \beta \rho[n, m]$. However, in practice the sensitivity functions tend to have small magnitudes towards the center of the image, making the constant sum assumption (and the corresponding SOS estimates) less accurate in this region. As a result, the use of the SOS estimates can produce aliasing artifacts at the center of the reconstructed image as illustrated in Figure 3(b). In the next section, we propose a new technique for estimating the sensitivity functions that does not rely on the SOS assumption.

\section{RECONSTRUCTION FRAMEWORK}

We first assume that the unknown sensitivity functions can be expressed in terms of a known basis expansion:

$$
s_{i}[n, m]=\sum_{q=0}^{R-1} \sum_{r=0}^{R-1} c_{i,(q, r)} \varphi_{(q, r)}[n, m],
$$

where

$$
\varphi_{(q, r)}[n, m]=n^{q} m^{r}
$$

are a set of polynomial basis functions, $R$ is the polynomial order in each dimension, and $c_{i,(q, r)}$ are coefficients associated with the $i$-th coil and the basis function $\varphi_{(q, r)}[n, m]$. Because the sensitivity functions are smooth, they can be well-represented by a basis of loworder polynomial functions [1],[6]. The basis characterization in (3) allows us to incorporate the prior knowledge of the smoothness of the sensitivity functions so that a unique solution can be determined. In other words, the number of unknowns becomes $R^{2} P+N M$, which is much smaller than the number of constraints $P N M$ when $R^{2} \ll N M$. In practice, we find that the sensitivity functions can be accurately represented using polynomials up to order $R=3$.

\subsection{Subspace-Based Framework}

Consider the pair of coils $i, h \in\{1,2, \ldots, P\}$. We define

$$
u_{i}[n, m] \stackrel{\text { def }}{=} \sum_{q=0}^{R-1} \sum_{r=0}^{R=1} d_{i,(q, r)} \varphi_{(q, r)}[n, m]
$$

to be the space of all functions formed from the basis functions $\varphi_{(q, r)}[n, m]$ in (4), and write the consistency relationship

$$
\rho_{h}[n, m] u_{i}[n, m]-\rho_{i}[n, m] u_{h}[n, m]=0 .
$$

Note that by (2), the consistency relationship (6) is satisfied for $u_{i}=$ $s_{i}$ and $u_{h}=s_{h}$. Expanding (6) using (2), we have

$$
\rho[n, m]\left(s_{h}[n, m] u_{i}[n, m]-s_{i}[n, m] u_{h}[n, m]\right)=0 .
$$

At pixels where the image function is nonzero $(\rho[n, m] \neq 0)$, the term within parentheses must be equal to zero. The multiplication of the polynomials in (7) can be viewed in terms of the 2-D convolution of the polynomial coefficients in (3) and (5), creating the equivalent condition:

$$
c_{h,(q, r)} * d_{i,(q, r)}-c_{i,(q, r)} * d_{h,(q, r)}=0,
$$

where $*$ denotes 2 -D convolution in $q$ and $r$. We note that this is equivalent to the condition in BMD methods such as EVAM that determine unknown FIR channel responses using a subspace framework $[4,5]$. Under the condition that the 2-D $z$-transforms of $c_{l,(q, r)}$, $l=i, h$, are coprime, the unqiue solution for $d_{l,(q, r)}, l=i, h$, satisfying (8) is [5]:

$$
d_{l,(q, r)}=\alpha c_{l,(q, r)},
$$

where $\alpha$ is a multiplicative constant common to both $i$ and $h$ (i.e., the solution is unique up to a scaling factor common to all channels).

\subsection{Estimation of Coil Sensitivities}

Using (5) and (6), and letting $k$ index the pair $(q, r)$, we obtain

$$
\sum_{k=1}^{K} d_{i, k} \psi_{h, k}[n, m]-\sum_{k=1}^{K} d_{h, k} \psi_{i, k}[n, m]=0
$$

where

$$
\psi_{i, k}[n, m]=\rho_{i}[n, m] \varphi_{k}[n, m]
$$

are known functions formed from the acquired low-resolution images and the polynomial basis functions, and $K=R^{2}$. Writing (10) in matrix notation produces

$$
\boldsymbol{A d}=\mathbf{0}
$$

where $\boldsymbol{A}=\left[\boldsymbol{\Psi}_{h} \mid-\boldsymbol{\Psi}_{i}\right], \boldsymbol{\Psi}_{i}=\left[\boldsymbol{\psi}_{i, 1}, \boldsymbol{\psi}_{i, 2}, \ldots, \boldsymbol{\psi}_{i, K}\right]$, $\boldsymbol{d}=\left[d_{i, 1} \ldots d_{i, K} \mid d_{h, 1} \ldots d_{h_{K}}\right]^{T}$, and $\boldsymbol{\psi}_{i, k}$ is a vectorized version 


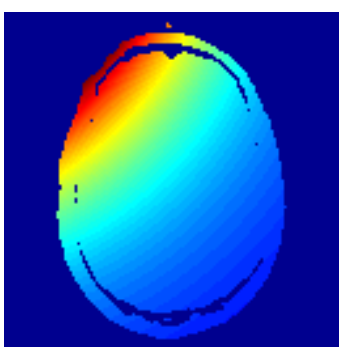

(a)

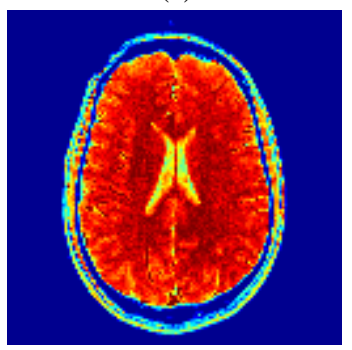

(e)

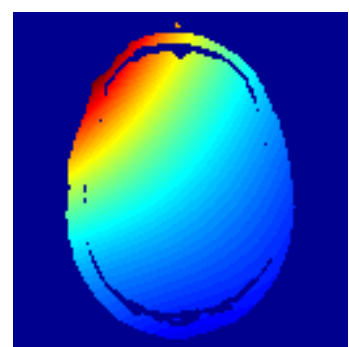

(b)

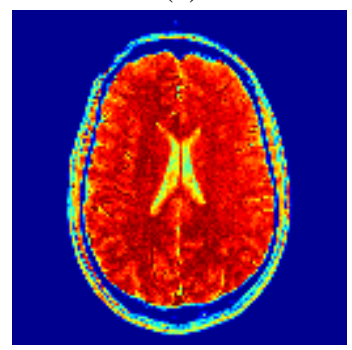

(f)

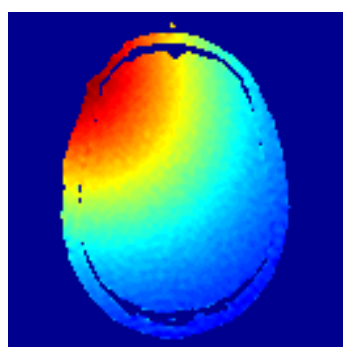

(c)

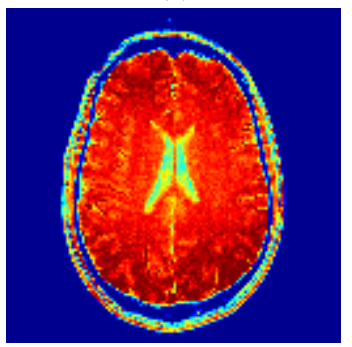

(g)

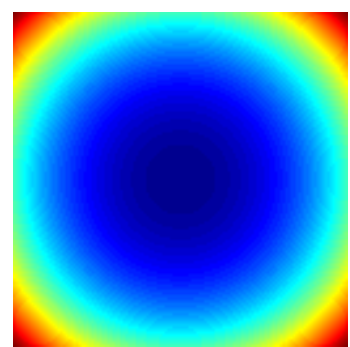

(d)

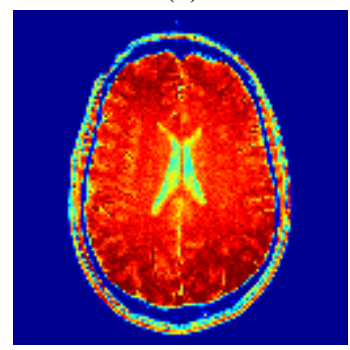

(h)

Fig. 2. Experiment using simulated sensitivity functions for an 8-channel head array coil, where the full-resolution image function is $N=128$ by $M=128$ pixels. An acceleration factor of $A=2$ is used along the $k_{y}$-dimension, and a $N^{\prime}=64$ by $M^{\prime}=64$ block of auto-calibration data is collected from each coil. In addition, additive white complex Gaussian noise with variance $N M(0.01 a)^{2}$ (where $a$ is the peak pixel magnitude) has been applied to the $k$-space data from each coil. (a) Applied sensitivity function for coil 4 , (b) estimated sensitivity function using the proposed technique, (c) SOS sensitivity estimate, (d) the root sum of the squares of all the sensitivity functions (note that this is not constant over the image), (e) original image, (f) reconstructed image using SENSE with sensitivity estimates from our method (SNR $=26.30$ $\mathrm{dB}),(\mathrm{g})$ SENSE reconstruction with the $\mathrm{SOS}$ sensitivity estimates $(\mathrm{SNR}=17.10 \mathrm{~dB})$, and $(\mathrm{h})$ GRAPPA reconstruction $(\mathrm{SNR}=17.06 \mathrm{~dB})$.

of $\psi_{i, k}[n, m]$. In this two-channel case, $\boldsymbol{A}$ is an $N M \times 2 K$ matrix, and by (9) the unique solution to (12) gives the coefficients of the unknown sensitivity functions: $\hat{\boldsymbol{d}}=\operatorname{Null}\{\boldsymbol{A}\}=\alpha \boldsymbol{c}$, where $\boldsymbol{c}=\left[c_{i, 1} \ldots c_{i, K} \mid c_{h, 1} \ldots c_{h_{K}}\right]^{T}$. In practice, the sensitivity functions may not be perfectly modeled by the assumed basis expansion, and additive noise may be present. Thus, $\boldsymbol{\Psi}$ generally has full column rank, and we use the least-squares solution given by the minimum right singular vector of $\boldsymbol{A}$.

\subsection{Efficient Estimation Procedure}

When there are more than two channels, $\boldsymbol{A}$ is constructed so that (10) is evaluated for every pair of channel responses simultaneously, similar to the block matrix structure used in the BMD techniques in [5] and [4]. This matrix can be prohibitively large to construct explicitly. However, the minimum right singular vector solution can be determined efficiently by determining the minimum eigenvector of the $P K \times P K$ matrix $\boldsymbol{B} \stackrel{\text { def }}{=} \boldsymbol{A}^{H} \boldsymbol{A}$. Due to the structure of $\boldsymbol{A}, \boldsymbol{B}$ can be determined directly without explicitly constructing $\boldsymbol{A}$. First, we form the matrices $\boldsymbol{\Psi}_{i}$ for $i=1,2, \ldots, P$. Given these matrices, $\boldsymbol{B}$ can be partitioned into $K \times K$ blocks $\boldsymbol{C}_{h, i}$ :

$$
\boldsymbol{C}_{h, i}= \begin{cases}\sum_{l \neq i} \boldsymbol{\Psi}_{l}^{H} \boldsymbol{\Psi}_{l} & i=h \\ -\boldsymbol{\Psi}_{h}^{H} \boldsymbol{\Psi}_{i} & i \neq h,\end{cases}
$$

where $i, h=1,2, \ldots, P$. Since $K P \ll N M$, the computational complexity of constructing $\boldsymbol{B}$ and computing its eigenvectors is $O\left(P^{2} K^{2} N M\right)$.

\subsection{Constructing the Basis Functions}

Thus far, we have assumed that $\rho[n, m] \neq 0$ in (7). However, outside of the object being imaged, $\rho[n, m]$ will be close to zero. In practice, the solution can be very sensitive to noise outside of the object. This is due to the basis functions in (4) having large values at the edges of the image, causing small noise values to be amplified in the product (11). Therefore, we apply a mask to each basis function in (4) to prevent noise outside of the object from being amplified. Such a mask can be determined by forming a composite image using SOS, determining the maximum value of this image, and thresholding out all values less than a small percentage of this value.

Applying a mask causes the basis functions to become approximately linearly dependent; this is due to the basis functions having support over a smaller window where the higher-order functions are well-approximated by combinations of lower-order terms. To improve the conditioning, we perform a Gram-Schmidt orthogonalization to the masked basis vectors.

\section{EXPERIMENTAL RESULTS}

Figure 2 presents an experiment using simulated sensitivity functions for an 8-channel head array coil, where the full-resolution image function is $N=128$ by $M=128$ pixels. To estimate the sensitivity functions, we applied our technique and SOS to the lowresolution images formed using $N^{\prime}=64$ by $M^{\prime}=64$ blocks of auto-calibration data from each coil. Figures 2(a)-(c) compare the actual and estimated sensitivities for coil 4 (selected as a representative example). The sensitivity estimate using our approach is observed to be more accurate than the SOS estimate. We observe sim- 


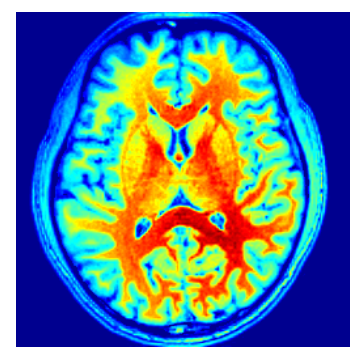

(a)

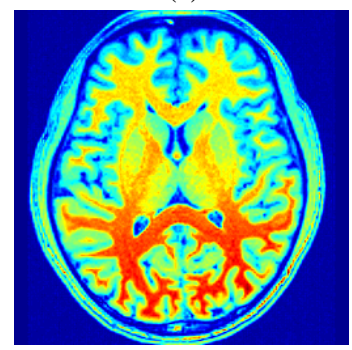

(c)

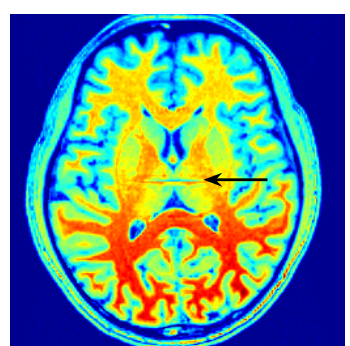

(b)

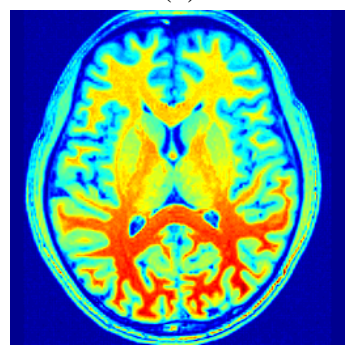

(d)
Fig. 3. Experiment using actual data from an 8-channel head array coil, where $A=2, N=M=256$, and $N^{\prime}=M^{\prime}=64$ : (a) SENSE image reconstruction using sensitivity estimates from the proposed technique, (b) SENSE reconstruction using SOS sensitivity estimates, (c) GRAPPA reconstruction, and (d) image reconstruction formed by applying SOS to the full $k$-space data where no subsampling is assumed $\left(N^{\prime}=M^{\prime}=256\right.$ and $\left.A=1\right)$. Note the aliasing artifact at the center of the SOS SENSE reconstruction in (b) (indicated by the arrow), which is due to wrong sensitivity estimates towards the image center; in the proposed reconstruction (a), such artifacts are reduced, and the contrast is improved towards the center of the image in comparison with (b)-(d).

ilar performance for the other coils. Figure 2(d) shows the root sum of the squares of the actual sensitivity functions; SOS assumes that the sum is constant over the entire image, but the figure reveals that this assumption is not accurate. Figures 2(e)-(h) compare the original image and reconstructed images using SENSE with the proposed technique, SENSE with SOS, and GRAPPA. In this experiment, the $k$-space data were subsampled using an acceleration factor of $A=2$ in the $k_{y}$ dimension. The contrast in the SOS SENSE and GRAPPA reconstructions is not as good as the reconstruction using our approach, and the SNR (measured with respect to the original image) is much lower. This is due to the SOS assumption being wrong towards the center of the image as shown in Figure 2(d), thus resulting in wrong image magnitudes at the center of the reconstructed images.

Figure 3 presents an experiment using actual data from an 8channel head array coil ${ }^{1}$. In this experiment, the full $k$-space data has dimensions $N=M=256$ and the auto-calibration data size is $N^{\prime}=M^{\prime}=64$. As in the previous experiment, we use an acceleration factor of $A=2$ in the $k_{y}$ dimension. Figures 3(a)-(c) compare the reconstructed images using SENSE with our approach, SENSE with SOS, and GRAPPA. The reconstruction using the proposed technique is observed to have much better contrast than the SOS SENSE and GRAPPA reconstructions towards the center of the

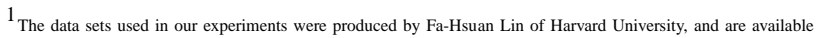
online at www.nmr.mgh. harvard.edu/ fhlin/tool_sense.htm
}

\begin{tabular}{c|c|c|c|}
$N^{\prime}, M^{\prime}$ & $A=2$ & $A=4$ & $A=8$ \\
\hline 32 & $21.68(11.94)$ & $10.71(9.32)$ & $6.48(-2.92)$ \\
\hline 64 & $22.17(12.05)$ & $10.87(10.60)$ & $6.82(-4.62)$ \\
\hline 128 & $27.98(12.08)$ & $11.29(11.82)$ & $7.12(-1.87)$ \\
\hline
\end{tabular}

Table 1. SNR $(\mathrm{dB})$ of the image reconstruction using the proposed technique versus acceleration factor and auto-calibration data size. The SNR using SOS estimates is shown in parentheses for comparison.

image. In particular, we observe that the SOS reconstruction has an artifact at the center of the image. This is an aliasing error due to wrong sensitivity estimates toward the center of the image. It comes from the edge of the brain being aliased to the center. For comparison, Figure 3(d) shows the reconstruction produced by SOS using the full $k$-space data from each coil $\left(N^{\prime}=M^{\prime}=256\right.$ and $\left.A=1\right)$. The result demonstrates that even in the best case where all of the $k$ space data are available, the SOS reconstruction has poorer contrast than the proposed reconstruction. In Table 1, we compare the reconstructed image quality using our approach under different acceleration factors $A$ and auto-calibration data sizes $N^{\prime}, M^{\prime}$. The SNR of the reconstructed images is measured with respect to a gold standard image formed by applying the proposed technique to the full $k$-space data from each coil (i.e., $N^{\prime}=M^{\prime}=256$ and $A=1$ ). The SNR of the reconstructions using the SOS estimates is also shown in parentheses for comparison. We observe that the performance under both approaches degrades as the acceleration factor increases.

\section{CONCLUSION}

We have proposed a novel subspace-based technique for estimating coil sensitivities in PMRI. Our method does not rely on the SOS assumption used in previous approaches, and thus avoids potential problems such as poor contrast and aliasing artifacts. Experimental results using actual and simulated data demonstrate improved performance in comparison with existing approaches.

\section{REFERENCES}

[1] K. P. Pruessmann, M. Weiger, M. B. Scheidegger, and P. Boesiger, "SENSE: Sensitvity encoding for fast MRI," Magnetic Resonance in Medicine, vol. 42, pp. 952-962, 1999.

[2] R. M. Heidemann, M. A. Griswold, A. Haase, and P. M. Jakob, "VD-AUTO-SMASH imaging," Magnetic Resonance in Medicine, vol. 45, pp. 1066-1074, 2001.

[3] M. A. Griswold, P. M. Jakob, R. M. Heidemann, M. Nittka, V. Jellus, J. Wang, B. Kiefer, and A. Haase, "Generalized autocalibrating partially parallel acquisitions (GRAPPA)," Magnetic Resonance in Medicine, vol. 47, pp. 1202-1210, 2002.

[4] M. Gurelli and C. Nikias, "EVAM: An eigenvector-based algorithm for multichannel blind deconvolution of input colored signals," IEEE Trans. on Signal Processing, vol. 43, no. 1, pp. 134-149, January 1995.

[5] G. Harikumar and Y. Bresler, "Blind restoration of images blurred by multiple filters: Theory and efficient algorithms," IEEE Trans. on Image Processing, vol. 8, no. 2, pp. 202-219, 1999.

[6] L. Ying, J. Sheng, and B. Liu, "Joint estimation of image and coil sensitivities in parallel MRI," in Proc. of the IEEE International Symposium on Biomedical Imaging, 2006, pp. 17-20. 